\title{
WAVEGUIDE MODES IN THE AKR SOURCE
}

\author{
I. Moiseenko*, M. Mogilevsky*, D. Chugunin*, \\ T. Romantsova*, and J. Hanasz ${ }^{\dagger}$
}

\begin{abstract}
The Auroral Kilometric Radiation (AKR) was investigated on measurements in the POLRAD experiment on the INTERBALL-2 satellite. It was revealed "lowfrequency" radiation with specific features in a spectrum on a polar edge of the auroral region. We have obtained that a series of narrow-band splashes are observed at frequencies between 35 and $70 \mathrm{kHz}$ with the period modulation some tens seconds and bow-shaped envelope. Possible interpretation of emission generation with a specific spectrum is discussed. We suppose that increase of intensity at small frequencies is interpreted as crossing by the satellite of the source region and observation of waveguide modes within it.
\end{abstract}

\section{Introduction}

The AKR is very intensive radio emission in a frequency range from $\sim 30$ to $\sim 700 \mathrm{kHz}$ with the peak intensity around $100-300 \mathrm{kHz}$. AKR is generated in regions with low plasma density near the local electron cyclotron frequency at altitudes between 2000 and 10000 $\mathrm{km}$ above the auroral region. The mechanism of AKR generation is the cyclotron maser instability [Wu and Lee, 1979] according to which in an unbounded medium the maximum radiation flux should be directed from the Earth at an angle $80^{\circ}-60^{\circ}$ in relation to a magnetic field. However the scheme of AKR generation in the limited source has been offered recently. It was accepted that the angle of radiation cone is defined by dimensions of the source which borders form a waveguide where waveguide modes propagate [Louarn and Le Queau, 1996; Burinskaya and Rauch, 2007]. The role of the source borders can play either a gradient of distribution of background plasma across an external magnetic field, or borders of an energetic electron beams, injected from magnetosphere tail into the internal region. Observations of AKR from the four-spacecraft Cluster have allowed to determine the AKR narrow angular beaming pattern [Mutel et al., 2008]. This implies that an observer located above the polar cap can detect AKR emission only from a small fraction of the auroral oval at a given location.

\footnotetext{
* Space Research Institue, Russian Academy of Science, 84/32 Profsoyuznaya Street, 117997, Moscow Russia

† Space Research Center, Polish Academy of Sciences, Toruń, Poland
} 
In this paper we show results of the LF-AKR measurements in the POLRAD experiment on the INTERBALL-2satellite [Hanasz et al., 1998] which can be interpreted as observation of waveguide modes in the generation source of radiation. Statistical observation of the LF-AKR have been described by Olsson et al. [2004], we consider certain cases in more details.

\section{Measurements and the Analysis}

In the present work we have analyzed measurements received from the POLRAD radiofrequency spectrometer in the frequency range from $4 \mathrm{kHz}$ to $1 \mathrm{MHz}$ since October, 1996 till February, 1997. During the given period the satellite moved for a long time along polar border of the auroral region. Spacecraft velocity in the direction to the pole $(\sim$ $50-200 \mathrm{~m} / \mathrm{s}$ ) was negligibly small in comparison with velocity of the polar border in the same direction $(\sim 7-10 \mathrm{~km} / \mathrm{s})$. For our analysis we have selected data including intervals of measurements near to the AKR source. A proximity to the source was defined on the lower frequency boundary of radiation - we chose those cases when lower frequency boundary was close to the local electron cyclotron frequency.

The bottom panel (Fig. 1) shows a quick - look spectrogram received on December, 1st, 1996 with the antenna $\mathrm{Y}^{\prime}$ (perpendicular to the spin axis of the spacecraft) in midnight sector of the magnetosphere. The horizontal time axis corresponds to 1 hour of data taking. The vertical frequency axis varies from $4 \mathrm{kHz}$ to $1 \mathrm{MHz}$. The spectral intensity of radiation is coded in black. The steady-state AKR in the frequency range $f=150$ $600 \mathrm{kHz}$ is observed during time interval from 16:14 to 16:45 UT. In this spectrogram the low-frequency (LF) narrow-band electromagnetic radiation occurs at frequencies $\sim 35-70$ $\mathrm{kHz}$ between 16:16 and 16:22 UT. An enlarged view of the LF - structure is presented in the upper panel (Fig.1). The horizontal time axis corresponds to 25 minutes and the vertical frequency axis spans the frequency range $4 \mathrm{kHz}-200 \mathrm{kHz}$. The frequencytime spectrum is divided into two parts by the electron gyrofrequency line (black dotted line).For the same period of time the local gyrofrequencies of electrons determined by the onboard magnetometer vary from 33 to $27,5 \mathrm{kHz}$. Below the gyrofrequency line VLF waves are observed (in our paper we don't consider such emissions). The LF - structure of 4 quasi-periodic splashes with bow-shaped envelope is observed above the gyrofrequency line during 6 minutes of measurements (16:16 -16:22 UT) between 35 and $70 \mathrm{kHz}$. These splashes have following features: 1) the characteristic period is $\sim 40-60 \mathrm{~s} ; 2$ ) the frequency width is $\sim 10-20 \mathrm{kHz}$. The first splash occurred at 16:16 UT at the frequency $\sim 50$ $\mathrm{kHz}$, when the altitude of the AKR source was $14620 \mathrm{~km}$ above Earth's surface. At that time the satellite was located at 23.6 MLT (Magnetic Local Time) and 71, $7^{0}$ invariant latitude; the altitude was $\sim 17216 \mathrm{~km}$ above Earth's surface. The subsequent splashes have been observed approximately at 16:17:20, 16:19:20 and 16:21 UT. During this time the spacecraft traveled $\sim 350 \mathrm{~km}$.

Figure 2 shows the AKR spectra in the frequency range from $4 \mathrm{kHz}$ to $300 \mathrm{kHz}$ for two cases (A - 27.01.97, B - 02.02.97). The signal below $20-30 \mathrm{kHz}$ is the whistler mode, which is not discussed in this paper. In two cases dotted curves (1) correspond to the steady-state AKR spectra in which decrease of the AKR intensity is observed at frequency decreasing 


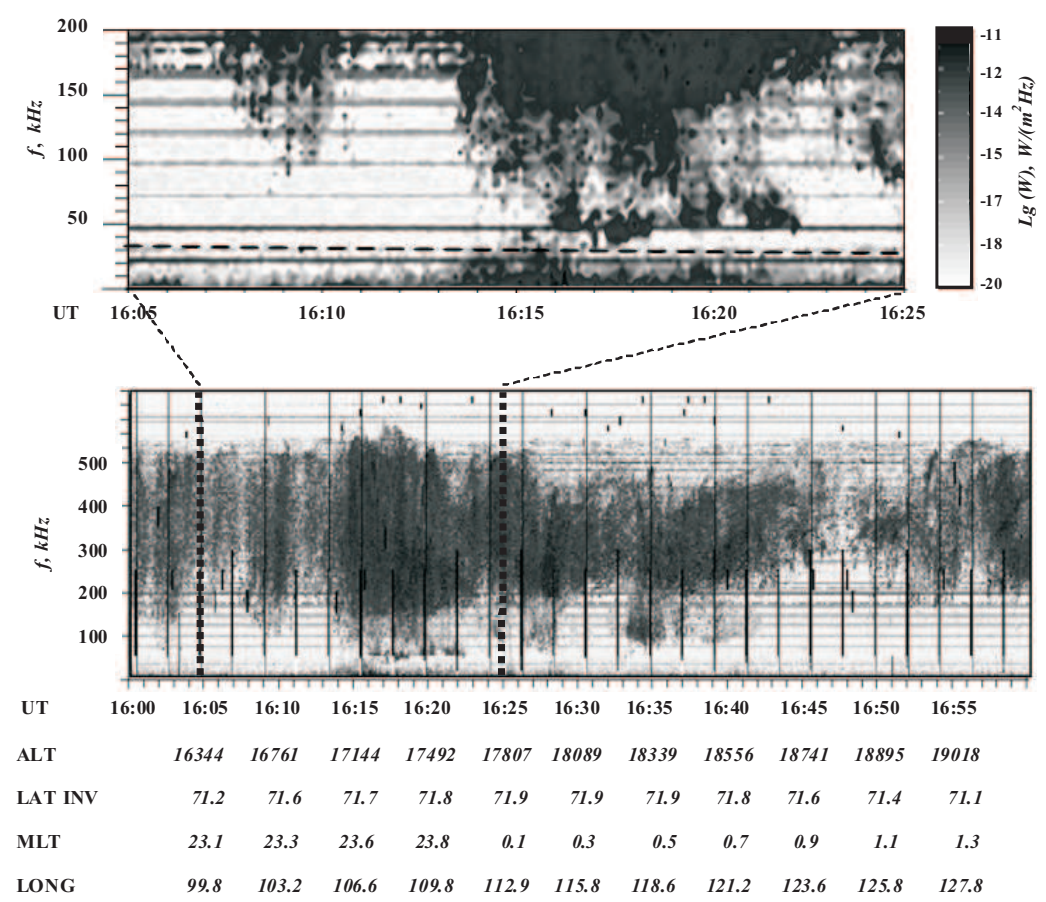

Figure 1: The dynamic srectrum of the AKR obtained with the POLRAD instrument on the 1st of December, 1996. A quick - look $1 \mathrm{~h}$ spectrogram received with the antenna $Y^{\prime}$ (bottom panel). Zoom of the same spectrogram showing the detailed spectrum in time interval from 16:05 to 16:25 UT and in the frequency range from $4 \mathrm{kHz}$ to $200 \mathrm{kHz}$ (upper panel). The dotted line is the electron gyrofrequency. The horizontal lines are interference lines.

(increasing of the signal at $25 \mathrm{kHz}, 50 \mathrm{kHz}, 75 \mathrm{kHz}$ etc are onboard interferences). When the low AKR frequency is close to the local gyrofrequency (the dotted vertical line) the signal increasing near low - frequency cutoff is observed (see solid curves (2)).

Figure 3 shows sketch of the AKR spectrograms near to the polar border of the auroral region, investigated in the given work. All considered spectra have been divided into 2 types (I type is presented on the left, II type - on the right). The spectrograms of I type were observed more often.

On the Fig.3 the horizontal axis corresponds to variation of time (UT) and the vertical axis - frequency change $(\mathrm{kHz})$. The steady-state AKR spectrum is presented by fill area, and the dotted curve is LF - radiation. The analysis of such spectra has shown that the difference between upper $\left(f_{1}\right)$ and lower $\left(f_{0}\right)$ frequencies of bow-shaped envelope makes $\Delta f \sim 10-20 \mathrm{kHz}$ that corresponds to height shift $\Delta H \sim 1500-3000 \mathrm{~km}$. 

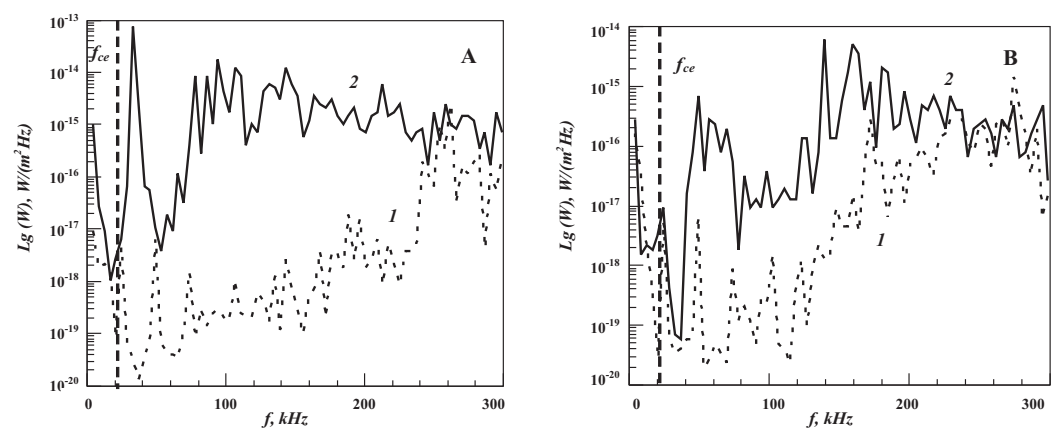

Figure 2: The AKR spectra in the frequency range from $4 \mathrm{kHz}$ to $300 \mathrm{kHz}$ for two cases (A 27.01.97, B - 02.02.97). In two cases dotted curves (1) represent the steady-state AKR spectra, solid curves (2) - the AKR spectra, when the LF - structures were observed. The dotted vertical line is the electron gyrofrequency.
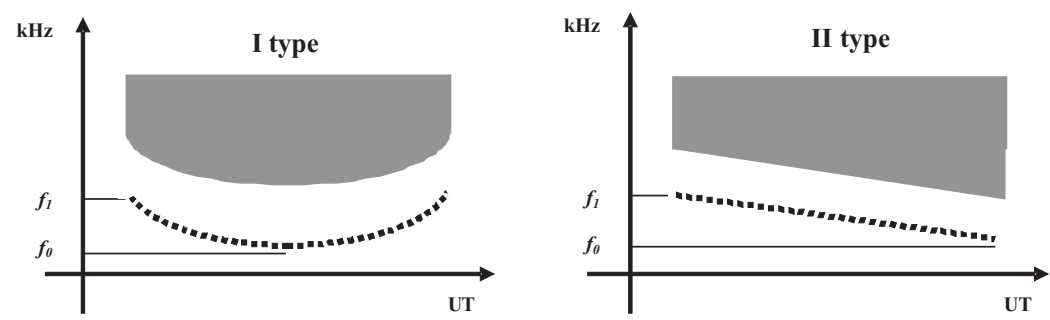

Figure 3: Sketch of the AKR spectrograms near to the polar border of the auroral region, observed on the INTERBALL-2 satellite.

Now we can summarize general observational results of radiation and discuss them further below. During expansion phase of geomagnetic substorms on the polar boundary of auroral zone narrowband LF - AKR with following characteristics was observed:

(1) The frequency width of splashes is $\sim 10-20 \mathrm{kHz}$.

(2) Signal increasing from antenna in $\sim 20$ - 70 times at low - frequency cutoff is observed.

(3) The difference between the lower frequency limits of LF - radiation and the local electron gyrofrequency is $\sim 8-20 \mathrm{kHz}$.

(4) Only in some cases amplitude modulation with a period of time $\sim 40$ - 60 s is observed. 


\section{Discussion}

The system of POLRAD antennas included two dipoles in total length of 22 meters that was much less than the wavelength of electromagnetic radiation in free space. In this case we can use the approximation of a short dipole. Figure 4 shows a comparison of the efficiency of such dipole during observation of long $\left(\lambda_{1}\right)$ and short-wavelength radiation $\left(\lambda_{2}\right)$.

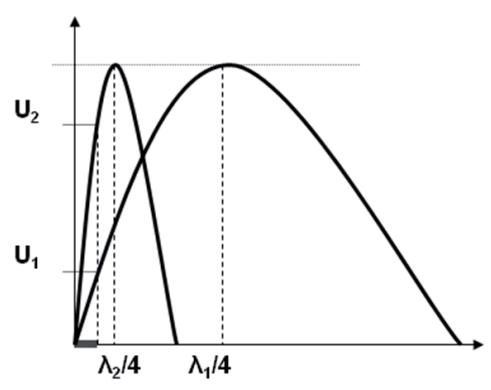

Figure 4: Sketch of the antenna efficiency. $\lambda_{1}$ is the AKR wavelength, $\lambda_{2}$ is the observed short-wavelength. $U_{1}$ and $U_{2}$ are induced potential on the antenna $\left(U_{2}>U_{1}\right)$.

The antenna is more efficient for short-wavelength emissions and it induces more potential than for the long-wavelength radiation $\left(U_{2}>U_{1}\right)$. Thus, a significant increase of the AKR intensity at low frequencies could be related to the observation of short-wavelength radiation. This situation is typical for wave propagation in the waveguide where the waveguide modes propagate more slowly than in free space.

The results of measurements on Viking satellite [Louarn and Le Queau, 1996] detected regions with low density of cold plasma, which indicate the existence of the waveguides extending along the magnetic field line (Fig. 5). The transverse width of such regions varies from a few kilometers to several tens of kilometers. These regions are filled by a hot and tenuous plasma $\left(n_{e} \ll 1 \mathrm{~cm}^{-3}\right)$, separated from the surrounding denser and colder plasma $\left(n_{e}>5 \mathrm{~cm}^{-3}\right)$ by sharp density gradients, which form waveguide borders. Also borders of an energetic electron beams (a few $\mathrm{keV}$ ), injected from magnetosphere tail into the auroral region can make waveguide borders.

Dimensions of the waveguide along the magnetic field line $(\Delta h)$ can be estimated by the difference between the lower frequency of AKR splashes and the local electron gyrofrequency, which in our experiment was $8-20 \mathrm{kHz}$. Tracing the satellite position along magnetic field lines at the height of the AKR generation was carried out using the CADR2 program [Galperin et al., 1980]. The results of calculations showed that the waveguide dimension along the magnetic field line is $1000-3000 \mathrm{~km}$.

Observation of amplitude modulation with the period of time $\sim 40$ - 60 s at low frequencies can be result of an interference inside the AKR source (waveguide) which borders are 


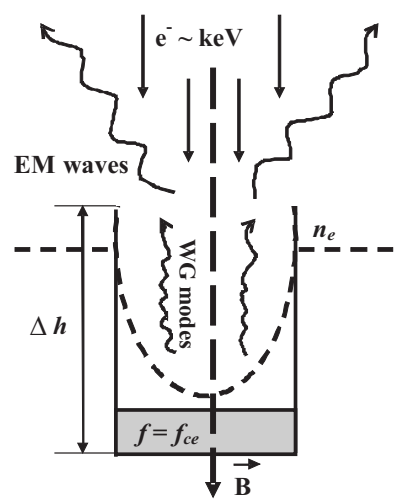

Figure 5: Sketch of the AKR limited source. Fill area $\left(f=f_{c e}\right)$ is the generation region, where the cyclotron maser instability develops. The dotted line $\left(n_{e}\right)$ is a density profile inside and outside the source. $B$ is a magnetic field line. Downright arrows are energetic electron beams (a few $\mathrm{keV}$ ), injected from magnetosphere tail. WG modes are waveguide modes, which propagate inside the AKR source. EM modes are electromagnetic radiation, which escapes from source. $\Delta \mathrm{h}$ is the waveguide size along the magnetic field line.

modulated by hydromagnetic waves (on condition that length of the hydromagnetic wavelength is longer than the wavelength of waveguide modes). On the other hand, amplitude modulation can be connected with a temporal effect, such as source amplitude oscillations. However simultaneous measurements of electron beams and optical observations have shown that the border of the auroral region passed through the satellite location only once. It implies that the satellite couldn't intercept source regions many times.

\section{Conclusion}

The AKR observations in the POLRAD experiment on the INTERBALL-2 satellite demonstrate waveguide modes in the source radiation. It implies that density distribution inside the AKR source is not uniform, inhomogeneities are extended along magnetic field lines and form the waveguide which defines characteristics of the diagram of the radiation. The waveguide size along the magnetic field line is several thousand kilometers that agrees quite well with earlier published theoretical models.

Acknowledgments. The authors are grateful to the referees for their constructive comments and remarks on an earlier version of this paper. This work was supported by the Russian Foundation for Basic Research (project no. 10-02-93115) and a Presidential grant for Support of Leading Scientific Schools (no. NSh-1739.2003.2). 


\section{References}

Burinskaya, T.M., and J.L. Rauch, Thin plasma cavities as a source of the Auroral kilometric radiation, in Planetary Radio Emissions VI, edited by H. O. Rucker, W. S. Kurth, and G. Mann, Austrian Academy of Sciences Press, Vienna, 241-248, 2006.

Galperin, Yu., Yu. Ponamarev, and V. Sinitsin, Some algorithms of calculation of the geophysical information along orbits of near-earth satellites, Preprint, SRI, 1980.

Hanasz, J., Z. Krawczyk, M. M. Mogilevsky, R. Schreiber, H. de Feraudy, K. Dudzinski, T. V. Romantsova, W. Nowakiewicz, A. Krayniuk, M. Barylko, A. Buczkowska, J. Juchniewicz, V. N. Nazarov, and N. Mikhalev, Observations of auroral kilometric radiation on board Interball-2: Polrad experiment (in Russian), Kosm. Issled., (English transl. Cosmic Res.), 36, 6, 56, 1998.

Louarn, P., and D. Le Quéau, Generation of the Auroral Kilometric Radiation in plasma cavities: - I. Experimental study, Planet. Space Sci., 44, 199-210, 1996a.

Louarn, P., and D. Le Quéau, Generation of the Auroral Kilometric Radiation in plasma cavities: - II. The cyclotron maser instability in small scale sources, Planet. Space Sci., 44, 211-224, 1996b.

Mutel, R. L., I. W. Christopher, and J. S. Pickett, Cluster multispacecraft determination of AKR angular beaming, Geophys. Res. Lett., 35, L07104, 2008.

Olsson, A., P. Janhunen, J. Hanasz, M. Mogilevsky, S. Perraut, and J. D. Menietti, Observational study of generation conditions of substorm-associated low-frequency AKR emissions, Ann. Geophys., 22, 3571-3582, 2004.

Wu, C. S., and L. C. Lee, A theory of terrestrial kilometric radiation, Astrophys. J., 230, 621-626, 1979. 
